\title{
Research on innovative personnel training mode of Interdisciplinary graduate
}

\author{
Keyong Shao \\ Scholl of Electrical Engineering \& Information \\ Northeast Petroleum University \\ Daqing, China \\ 1783811235@qq.com
}

Weijian Ren

Scholl of Electrical Engineering \& Information Northeast Petroleum University

Daqing, China

1064619284@qq.com

\author{
Huizhen Zhang \\ Scholl of Electrical Engineering \& Information \\ Northeast Petroleum University \\ Daqing, China \\ 149004028@qq.com
}

\author{
Li Yang \\ Scholl of Electrical Engineering \& Information \\ Northeast Petroleum University \\ Daqing, China \\ 19696163@qq.com
}

\begin{abstract}
Interdisciplinary graduate education is conducive to optimizing the knowledge structure of graduate students for fostering graduate creative ability, to strengthen the discipline construction of graduate education for fostering the cultivation of innovative talents. At present our country university interdisciplinary graduate education are facing some of the urgent need to overcome the practical difficulties, such as in academics organization structures, the management and training systems of institutions of higher learning. Based on the above contents, we propose the following methods to promote interdisciplinary graduate education, including the optimization of curriculum system for graduate students, innovation of graduate education management system, and construction multidisciplinary academic exchange platform and strengthen quality assessment management.
\end{abstract}

Keywords- interdisciplinary education; graduate education; cultivation of innovative talents

\section{INTRODUCTION}

Since twentieth Century, the development of science and technology more and more shows the trends of multidisciplinary intersecting, mutual penetration, systematization and integration. At present, with the rapid expansion of the scale of graduate education and more and more kinds of postgraduate training, existing single subject training mode cannot meet the needs of graduate education reform and development. So we should construct the culture environment and mechanism of cross discipline, and implement interdisciplinary education, cultivate interdisciplinary talents to solve the comprehensive social problems in the future, and this has become the consensus and the tendency for the development of graduate education, but also an inevitable choice for postgraduate education reform and development.
In order to realize the goal of education is necessary to the implementation of cross disciplinary training of graduate students. One of the purposes of education should be to make the educators fully developed both in intelligence and ability based on the meaning of the comprehensive development. Interdisciplinary is consistent with the basic principles of pedagogy, pedagogical knowledge and ability with the uniform principles, associative principles and the principle of combining theory with practice match. In addition, interdisciplinary degree programs are beneficial to $t$ the construction and development of this special subject. If there is a considerable gap between domestic doctoral training and doctoral training in developed countries, then the inadequacies of our country's training is lack of information and narrow range of knowledge. Exploring cross-disciplinary training mode is a high level of education to adapt to both technological development trends and the implementation of the urgent requirement of our education strategy.

\section{INTERDISCIPLINARY GRADUATE EDUCATION BENIFITS THE CULTIVATION OF INNOVATIVE TALENTS}

We must have a large number of creative talents for support to build an innovation-oriented country. Graduate students is the highest academic degree in the education system and the main way to train and bring up creative talents. The graduate student stage is an important station to one's scientific career. The graduate students are full of energy and their thoughts are active, which is the best age to generate new ideas and new results. Therefore, the graduate education is a high-level teaching and talent training activity. Its teaching content is the academic development of cuttingedge knowledge. Its mean of cultivating is scientific research.And its learning goal is the innovation of the knowledge. "The core of postgraduate education is the 
cultivation of innovation ability". Relative to the undergraduates, the graduate student's physical and mental development has reached the requirement to go to a higher level of study. At the same time, tutor itself is of the deepest academic attainments and strongest scientific research ability in the universities internal to engaged in high-level talents training and knowledge innovation. For this reason, the graduate student education must take the mission to knowledge innovation, technological innovation and cultivate high-level talents with innovation spirit and innovation ability. Of course, different people have different views on how to cultivate the graduate student's innovation ability. But we believe that the interdisciplinary graduate education is indispensable to cultivate innovative talents under the background of the modern knowledge economy. Because the innovation of every subject largely depends on the exchange with the professional fields belong to other subjects.

\section{A. Interdisciplinary graduate education helps to improve the structure of the graduate student's knowledge}

The salient feature of top creative talents is that they have the solid theory foundation of basic skills and reasonable knowledge structure and it also requires multi-disciplinary comprehensive knowledge at the same time. A single discipline knowledge structure has been difficult to solve the increasingly sophisticated technology and practical problem. We can talk about innovation only when we have the multidisciplinary knowledge and the ability of integration, analysis, and reconstruction of the knowledge structure. Interdisciplinary education forms a new discipline through the mutual fusion, mutual infiltration and interaction of the knowledge of different disciplines. Or it is a knowledge system to solve a problem as the center. It is advantageous to the postgraduate students to form a background with multidisciplinary knowledge, to overcome the narrowness and oneness of original knowledge, and to construct a knowledge structure with the relative integrity, comprehensive and its multi-discipline. Consequently it urges graduate mining their development potential and enhance their comprehensive quality and creative ability.

\section{B. Interdisciplinary education helps to train the innovative ability of graduate students}

The key to develop the innovative ability of graduate students is the constantly improve of the creative thinking ability and the innovation of scientific research method. Interdisciplinary research is the process of researchers in different disciplines background makes different disciplines knowledge combined with each other by using the correlation, compatibility and complementarities between different disciplines and to promote knowledge innovation in the multidisciplinary integration, interaction and multidirectional communication. Interdisciplinary education helps to cultivate the graduate to form the cross thinking ability and this is an important part of the creative thinking ability. At the same time, it can help the graduate students get rid of the bondage of fixed single subject thinking mode , absorb the nutrition of the thinking mode of other disciplines and integrate the specific thinking mode of two or more disciplines organically by guiding graduate students to engage in interdisciplinary research to realize the innovation of the mode of thinking. Interdisciplinary education can not only make the graduate students to master the principle and method of relevant disciplines, can also improve and complete the traditional technology innovation methods and promote the cultivation of innovative talents.

\section{THE PRACTICE DILEMMA OF CROSS-DISCIPLINARY POSTGRADUATE EDUCATION TRAINS INNOVATIVE TALENTS}

\section{A. Disorder of discipline organization system}

First, differences of the subject organizational culture and behavior orientation. Each subject has its own unique discipline culture, and its difference is embodied in the difference of discipline organization goals. A academic department form their own department of culture through traditional and historical precipitation, which gets the members together and shares a common value. When a college is required to carry out an interdisciplinary research among different disciplines, this kind of cultural value difference becomes obstacles to communication and cooperation. Second, the discipline organization structural barriers. Discipline organization in the university is mainly the college, department and institute, these traditional academic organizations occupy the mainstream status in the university and have formed a relatively stable university organizational structure. The structure resistance of interdisciplinary research comes from that the modern university pays great attention to implement hierarchical organizational structure, which attaches great importance to the longitudinal hierarchy communication and the high efficiency of communication, however, discipline organization is around the logical structure of the discipline development, especially the interdisciplinary research and interdisciplinary graduate education should put more emphasis on horizontal communication between discipline organization in theory, it also led to the isolation and differentiation between discipline organization and lack of communication and coordination between different discipline organization. Third, the conflict of the role and identity of the teacher. In the interdisciplinary research organization, with the role and identity of the disciplinary organization, researchers enter into the interdisciplinary research organization in which they have another role and identity, two kinds of different role and identity led to the conflict; At the same time, in the interdisciplinary research organization, members are often teachers and researchers from different subject. They have different discipline culture, organization beliefs and they have different role and identity in their original discipline organizations, which form the conflict, in the interdisciplinary research organization, this conflict is during the whole research process, it is the basic contradiction of the cross study, the role and identity conflict in fact contains a deep subject culture conflict. 


\section{B. The Disorders of University management system}

First, faculties based ingrained, college barriers rugged, it's difficult to realize resource sharing. Although giving a certain degree of attention interdisciplinary graduate training , at the practical level, each college not really formed the situation integration of resources, sharing of resources, information interaction, objective of interdisciplinary graduate training overall lack of grasp. This increased interdisciplinary research and teaching difficult, is not conducive to the cultivation of innovative talents. Second, the management system lag, training programs are too rigid. For a long time, postgraduate training model of a single country, ignoring the disciplines required individual research capabilities and other personalized factors. In addition, the required training program required courses too much, and elective courses is too small, the range of interdisciplinary graduate course is narrow. Third, disciplines unreasonable. The readjustment and the range of professional schools are limited, survival and interdisciplinary professional development opportunities, space has been greatly restricted. Forth, teachers of different disciplines collaborate on student training are lack of effective. Interdisciplinary implement innovative talents will inevitably require the students to choose a professional, courses and teachers scope. But in practice, teachers only focus on the subject areas of expertise and the students research and culture, there is no resource sharing mechanisms. This discipline professional setting mode also resulted disciplines and teaching resources division, forming a fortified barrier professional disciplines, but also a lack of communication and exchange between disciplines. Promote it restricts the fundamental disciplines to break boundaries, resource sharing mechanism, students free choice of profession as a necessary condition for interdisciplinary training innovative talents.

\section{Creating academic atmosphere and building a variety of forms of interdisciplinary academic exchange platform}

Interdisciplinary academic communication is not only promote the fusion of interdisciplinary, produce spark and form new opinions of effective channel, but also an integral part of interdisciplinary graduate education form. Therefore, colleges and universities must break through the limited to the professional communication set of academic issues, build diverse interdisciplinary academic exchange platform. First of all, colleges and universities should actively strengthen with universities, scientific research, cooperation between enterprises and other units, regularly carry out across industry sectors and geographic regions, international graduate student academic exchange activities, to encourage graduate students to actively participate in and gradually institutionalized. Second, the school should build various types and various levels and various forms of interdisciplinary academic exchange platform. From the type, can hold the interdisciplinary academic seminar, can also organize the interdisciplinary graduate tutor BBS, outside experts BBS and graduate students ect. From the level that can be held within a school cross major, interdisciplinary academic BBS, can also be a few college to hold together the interdisciplinary academic BBS, can also be organized by the school wide range of interdisciplinary academic theory.

\section{Strengthen the graduate education of quality assess- ment management, improve the quality of graduate education evaluation system}

The formation of creative ability through out every link in the interdisciplinary training of graduate students, thus enhancing the quality of postgraduate education management plays an important role in improving its innovation ability. Development of a late start to assess the quality of graduate education in China, and as a result of China's higher education centralized management system for a long time, and gradually formed a single government administration based on the assessment of the quality of graduate education evaluation system. As an important part of self evaluation and social evaluation has not been really set up. Therefore, China can draw lessons from the foreign advanced experience, and fully consider the impact of China's specific national conditions and cultural tradition, establish the Chinese graduate education quality evaluation system.

- Strengthening internal management training for quality assessment of all aspects of graduate students. Training quality evaluation as a regular activity of graduate students, all colleges and universities should be regularly carried out self evaluation. Graduate quality management including recruitment, training. All aspects of the thesis defense, degree granting, ideological and political education, employment guidance, including all aspects of teacher's team construction, subject construction, teaching environment construction. Therefore, colleges and universities should ensure in all links must be established scientific and reasonable management system, a clear assessment standard, accelerating the construction of interdisciplinary research quality self assessment system of postgraduate training.

- To realize the diversification of interdisciplinary graduate education quality evaluation. In China, education research of the evaluation work which is mainly by the education department in charge of graduate cultivation units for evaluation, evaluation activities have a lot of administrative functions, and it can not achieve the quality assessment due social effect. Therefore, the foundation is necessary in the internal evaluation, external evaluation mechanism, to establish reflect social diversity requirements agency. In the assessment of the main body, change a subject should be changed from single government to government, universities, social pluralistic direction, and gradually establish a government, universities and society education quality three-inone graduate student evaluation system.

- Strengthen interdisciplinary graduate education evaluation theory and building assessment team is necessary. Quality assurance of graduate education is a complex social activity. To make this activity with direction and scientifically correct, not only 
have to guarantee the quality of our practice as the source, but also have a corresponding theoretical basis. In addition, evaluation personnel specialized degree is to improve the evaluation of professional, the evaluation of the authority and the social recognition of the important safeguard. It not only avoid the deviation in evaluation work, but also improve the efficiency and effectiveness of the effective guarantee.

\section{CONCLUSION}

Interdisciplinary graduate student innovation ability training is not only refers to the intersection of transverse sense, should also contain the meaning of longitudinal covering, Pay attention to basic research, applied research and development and research of cross . Interdisciplinary graduate student innovation ability training in our country is still at the exploratory stage, is a supplement of the graduate education system model, It with the traditional sense of the subject graduate training model does not conflict

In addition, the interdisciplinary graduate student innovation ability is a complicated system engineering, Is a need to improve the work, Need further quantification, standardization, theory elaboration, let the interdisciplinary graduate innovation theory are no longer words, rhetoric, but can form a set of operations, can implement specific solutions, and constantly improve the theory is applied to all aspects of the graduate student work, only in this way, to improve graduate student innovation ability.

\section{ACKNOWLEDGMENT}

This research was financially supported by the Key Topic of Heilongjiang Province of China(Grant NO.GBB1212018) and the reform project of degree and postgraduate education in Heilongjiang Province of China(Grant NO.JGXM_HLJ_ 2012023)

\section{REFERENCES}

[1] Wang Xiaoli, Xu Xiaowen. The cultivation of innovation ability is the core of Graduate Education[J]. Research on Higher Engineering Education,2002,(3):64-66.

[2] Zhou Yezhong. Reflections on the cultivation of Interdisciplinary Graduate[J]. China Academic Journal Electronic Publishing House, 2007,(8):11-15.

[3] Liu Chujia. The development of universities interdisciplines[J]. Institutions of Higher Education,2002(6):76-79.

[4] Xiang Peili. Interdisciplinary research: Education problem demanding prompt solution on graduate students[J]. Journal of Guangxi University(Philosophy and Social Science),2003,(1):95-98.

[5] Qian Peisi, Li Junjie. The establishment and operation of university interdisciplinary education organization[J]. Journal of Zhejiang University of Technology (Social Science),2005,(2):166-169. 